\title{
Serodiagnosis of Tuberculosis: Specific Detection of Free and Complex-Dissociated Antibodies Anti- Mycobacterium tuberculosis Recombinant Antigens
}

\author{
María Susana Imaz', María Fernanda Schmelling1, Susanne Kaempfer², Ralph Spallek ${ }^{2}$ and Mahavir Singh ${ }^{2,3}$ \\ ${ }^{1}$ National Institute of Respiratory Diseases Emilio Coni; Santa Fe, Argentina; ${ }^{2}$ Lionex GmbH, Braunschweig; ${ }^{3}$ Department of Genome Analysis, \\ Helmholtz Center for Infection Disease; Braunschweig, Germany
}

\begin{abstract}
The diagnostic test characteristics of detecting free and complex-dissociated IgG to three recombinant antigens of Mycobacterium tuberculosis (38-kDa, Ag16 and Ag85B), singly and in combination, were evaluated in sera from 161 tuberculous patients [smear-positive pulmonary TB (50), smear-negative pulmonary TB (pTBsm-) (60) and extrapulmonary TB (51)) and 214 control patients (mycobacteriosis (14), mycoses(14), leprosy(4), other underlying diseases (82) and healthy people (100)]. The individual antigens ranged from $25 \%$ to $42 \%$ in sensitivity and from $\mathbf{9 3 \%}$ to $\mathbf{9 6 \%}$ in specificity, while considering free IgG response. Addition of complex-dissociated antibodies against each individual antigen improved the sensitivity up to $55 \%$. The number and levels of specific antibodies varied greatly from individual to individual. Combination of individual results for free and complex-dissociated IgG to 38 kDa, Ag16 and Ag85B offered $76 \%$ sensitivity and $83 \%$ specificity. When the three antigens were placed in the same well, the sensitivity was lower than that expected on the basis of single antigen $(63 \%)$ but with a good specificity (95\%), even in the group of mycobacteriosis or mycoses. The highest contribution of complex-dissociated IgG results to free IgG results was seen for the diagnosis of pTBsm- patients. In conclusion, although neither single recombinant antigen was reactive with most sera from TB patients even after the measurement of both free and complex-dissociated antibodies, the use of multi-antigen cocktails improved the diagnostic utility of the ELISA assay, allowing the identification of almost $70 \%$ of pTBsm-, with a high level of specificity; the use of additional, well selected antigens should lead to the detection of almost all patients with TB.
\end{abstract}

Key-Words:

To control tuberculosis (TB), it is still necessary to find diagnostic methods that are both more rapid to carry out and more sensitive than traditional methods (smear and culture) but which are simpler and less expensive than the new molecular diagnostic tests that are based on the amplification of nucleic acids. Serological methods, which seem to be the ideal choice, are usually based on the detection of free soluble circulating antibodies against mycobacterial antigens. Research in the identification of a suitable antigen for serodiagnosis of TB has come a long way, yet there is a lot more to be explored [1,2]. In fact, no serological test is commonly used in the diagnosis of TB. One of the main reasons for the lack of a routine test is the variability and often low sensitivity and specificity of the tested antigens. Specificity has been increased by using purified antigens but unfortunately at the expense of loss of sensitivity $[1,3]$. On the other hand, the antibody repertoire in TB is highly diverse, with sera from different persons reacting with different antigens, reflecting the fact that accurate serodiagnosis of TB possibly requires multiple antigens to cover the breadth of the antibody response [3]. However, independent of the single-antigen or combination of antigens used, a percentage of patients with well documented TB still present low levels or even absence of specific antibody against any of the

Received on 28 November 2007; revised 22 March 2008.

Address for correspondence: Dr María Susana Imaz. Instituto Nacional de Enfermedades Respiratorias "Emilio Coni”. Blas Parera 8260. Santa Fe (3000) Argentina. Phone/Fax: 54-342-489-2830. E-mail: suimaz@yahoo.com; rfernand@fcjs.unl.edu.ar.

The Brazilian Journal of Infectious Diseases 2008;12(3):234-244. (C) 2008 by The Brazilian Journal of Infectious Diseases and Contexto Publishing. All rights reserved. antigens evaluated. Such false negative results in patients with TB could be explained by an absence of detection by regular ELISA owing to the formation of immune complexes (IC), as previously reported [4-6].

We therefore looked at measuring the level of free and complexed IgG to antigens that previously demonstrated an ability to induce humoral responses in human TB by focusing on molecules suggested to possess species-specific serodominant epitopes. Daniel and Anderson [7], were the first to report on the species-specific antigen 5 of Mycobacterium tuberculosis, which was later proved to be the same as the 38-kDa protein [8]. This antigen has shown high specificity in all studies conducted in different parts of the world (88\% to 98\%) [1,2]. However, sensitivity varied from 49 to $89 \%$, being higher in populations where more patients present with advance disease [9-15]. Other than this, the immunodominant 16-kDa antigen (Ag16) of M. tuberculosis, a molecule homologous with proteins belonging to the family of low molecular-weight heat shock proteins [16], also contains B-cell epitopes specific for $M$. tuberculosis complex. As in the case of the 38-kDa antigen, it has been suggested that its species-specific (restriction) antigenicity of mycobacterial species almost certainly rests not with the whole antigen but with their epitopes [17]. Furthermore, it has been suggested that Ag16 is immunogenic in the early stages of infection of $M$. tuberculosis and in primary TB $[1,18]$. The Ag85 protein complex consists of at least three distinct but related proteins, designated as $85 \mathrm{~A}, 85 \mathrm{~B}$, and $85 \mathrm{C}$, as shown by crossed-immunoelectrophoresis. This antigen has been varyingly named in literature as a2, alpha antigen, fibronectinbinding protein or antigen 6 and 85 complex [19]. Interestingly, positive antibody titers were more frequent in non-cavitary 
tuberculosis [20,21] and, although homologues of Ag85 have been reported to be present in non-pathogenic mycobacteria and in corynebacteria [22], the presence of M. tuberculosis complex-specific serodominant epitopes has been suggested [20]. Of the three components, 85B has been found to have superior value for diagnosis [23,24].

Furthermore, in order to develop specific serodiagnostic reagents, substantial amounts of mycobacterial antigens are required. Purification of such antigens directly from $M$. tuberculosis is difficult because of low cell yield, low growth rates and the virulent nature of the organisms. A potential solution to this problem is the production of recombinant antigens in organisms such as Escherichia coli (E. coli).

On the other hand, many authors has pointed out that the profile of antigens recognized by patient antibodies is influenced by the stage of TB $[25,26]$, suggesting that valid comparison of potential serodiagnostic antigens can be made only if the same cohort is used for assessment of the different candidate antigens under study. Therefore, the purpose of this study was to evaluate the diagnostic potential of measuring free and complex-dissociated antibodies against three previously characterized recombinant proteins of $M$. tuberculosis (38-kDa antigen, Ag16 and Ag85B) in the sera of TB patients and various control subgroups. The recombinant antigens were not only used individually, but were combined in the same microplate well, in order to evaluate the possibility of providing more accurate tools for immunologic diagnosis.

\section{Material and Methods \\ Patients Sera}

A total of 375 serum samples were obtained from the following groups of subjects.

\section{Tuberculous Patients}

One hundred and sixty one serum samples from human immunodeficiency virus-seronegative individuals (age range, 16 to 70 years, percentage of males, $60 \%$ ) were studied. All these patients were investigated for TB at the National Institute of Respiratory Diseases "Emilio Coni” in Santa Fe, Argentine. One hundred and ten serum samples were from patients with pulmonary TB (pTB), and 51 serum samples were from patients with extrapulmonary TB (epTB). The pTB group consisted of 50 smear-positive and culture-positive patients (pTBsm + ) who had active lesions on chest radiograms and of 60 patients from whom two consecutive tests on admission were smear-negative (pTBsm-); 47 of them were culture-positive and with active chest radiogram lesions, and 13 patients with culture-negative tuberculosis who had been diagnosed by suggestive clinical and radiological findings with marked improvement after antituberculosis treatment. The extrapulmonary locations involved pleurisy (28 patients), urinary tract (5 patients), meningitis (4 patients), disseminated locations (5 patients), lymphatic tissues (4 patients), peritonitis (2 patients), pericarditis (2 patients) and osteitis (1 patient).
The patients had not yet started antituberculosis treatment when the serum samples were taken.

\section{Control Subjects}

Two hundred and fourteen serum samples from human immunodeficiency virus-seronegative individuals were included as controls. They were divided as follows:

a. Patients with pulmonary mycobacteriosis and mycoses. Consisting of 28 patients (age range, 23 to 61 years; percentage of males, 58\%) with pulmonary diseases usually considered in the differential diagnosis of TB like pulmonary mycobacteriosis (14 patients) and mycoses (14 patients). The patients were included as follows: (i) pulmonary diseases produced by nontuberculous mycobacteria (NTM) (Mycobacterium avium complex, 10 subjects; Mycobacterium chelonae, 2 subjects; Mycobacterium fortuitum, 2 subjects), ii) pulmonary mycoses (paracoccidioidomycosis, 10 subjects; aspergillosis, 2 subjects; histoplasmosis, 2 subjects).

b. Patients with underlying disorders other than mycobacteriosis and mycoses (Others). This group was composed of patients with suspected tuberculosis, in whom a firm alternative diagnosis was made. Consisted of 82 patients (age range, 16 to 78 years, percentage of males, 54\%) who were being treated for underlying disorders other than mycobacteriosis and mycoses, including pneumonia, 48 subjects; lung cancer, 2 subjects; asthma, 2 subjects and nonrespiratory diseases, 30 subjects (nephrotic syndrome, 6 subjects; staphylococcal abscess, 2 subjects; congestive hearth failure, 11 subjects; acute bacterial meningitis, 11 subjects). Additionally, all controls with respiratory symptoms were assessed by X-Ray; those with productive cough were bacteriologically evaluated. Seventy six subjects had Mycobacterium bovis BCG vaccination history. The purified protein derivative (PPD) status of the patients was unknown. In many countries, such as Argentina, the PPD test for diagnosis of adult TB is considered difficult to interpret and, therefore, suspected TB is not usually evaluated by tuberculin skin test in routine clinical practice $[27,28]$.

c. Clinically healthy patients (CHP). Consisted of 100 apparently healthy persons (age range, 15 to 69 years, percentage of males, 51\%) who agreed to undergo routine analysis examinations at a laboratory. All of them showed normal findings in the blood and clinical examinations. Ninety five subjects had Mycobacterium bovis BCG vaccination history.

d. Leprosy patients. This group included four cases of leprosy (age range, 36 to 46, percentage of males, 75\%).

\section{Mycobacterial Antigens}

Culture filtrate antigens (CFA) of $M$. tuberculosis were obtained as previously described [29]. Briefly, M tuberculosis 
H37Rv was grown for 8 weeks in Dorset Henley's medium. Bacteria were killed by treating the suspension with $1 \%$ phenol at $37^{\circ} \mathrm{C}$ for 3 days. Culture was filtered through cellulose asbestos membranes. The filtrate was then precipitated with trichloroacetic acid (4\%) and dissolved in a phosphate buffer $\mathrm{pH} 8$ at a concentration of $2 \mathrm{mg} / \mathrm{mL}$. Recombinant mycobacterial antigens 38-kDa, Ag16 and Ag85B were supplied by Lionex GmbH, Germany. They were expressed as His-tagged proteins in E. coli from the complete coding sequences and purified by subsequent Ni-affinity chromatography [30].

\section{Enzyme-Immunoassay Microplate Preparation}

Microtiter plates (Immulon 2, Dynatech Laboratories Inc., Chantilly VA, USA) were coated with recombinant mycobacterial antigens or CFA diluted to a concentration of $2 \mu \mathrm{g} / \mathrm{mL}$ (Ag85B, 38-kDa, Ag16) and $25 \mu \mathrm{g} / \mathrm{mL}$ (CFA) in $0.05 \mathrm{M}$ carbonate buffer, $\mathrm{pH} 9.6$, and incubated overnight at $4^{\circ} \mathrm{C}$. After coating, the reactive sites were blocked by incubation for $1 \mathrm{~h}$ at $37^{\circ} \mathrm{C}$ with phosphate buffer saline (PBS) containing $1 \%$ bovine serum albumin (BSA).

\section{Preparation of Complex-Dissociated Antibodies}

Dissociation of IC was done as previously described [31]. Briefly, to $100 \mu \mathrm{L}$ serum $100 \mu \mathrm{L}$ PBS (1:1) was added. An equal volume $(200 \mu \mathrm{L})$ of $5 \%$ polyethylene glycol 6000 (PEG 6000) in PBS (PEG-PBS) was added and the mixture was incubated at $4^{\circ} \mathrm{C}$ overnight. After centrifugation at $1500 \mathrm{~g}$ for $20 \mathrm{~min}$, the pellet was washed twice with 2.5\% PEG-PBS solution. The pellet was then resuspended in $200 \mu \mathrm{L}$ in PBS with $10 \mathrm{mM}$ EDTA at $\mathrm{pH} 7.4$ and incubated at $37^{\circ} \mathrm{C}$ for $30 \mathrm{~min}$; then $600 \mu \mathrm{L}$ of cold 0.2M Glycine-HCl (pH 6.8) was added. After incubation for $15 \mathrm{~min}$ at $4^{\circ} \mathrm{C}$, the solution was neutralized by adding $50 \mu \mathrm{L}$ of $1 \mathrm{M} \mathrm{K}_{2} \mathrm{HPO}_{4} \mathrm{pH} 9$ and diluted with $2.35 \mathrm{~mL}$ of PBS containing $1 \%$ BSA, giving a final dilution of 1:32. Samples were used within $30 \mathrm{~min}$.

\section{Enzyme-Immunoassay}

\section{ELISA with Recombinant Antigens}

Samples tested for free antibodies from plain serum were diluted 1:100 in 1\% BSA-PBS with the addition of $0.05 \%$ Tween-20, whereas for IC-dissociated antibodies, the samples were diluted to 1:32 in BSA-PBS without Tween-20 as explained before (Section Preparation of complex-dissociated antibodies). One hundred $\mu \mathrm{L}$ of each sample was placed in the respective microplate well sensitized with the antigen for free antibodies and, with and without antigen for ICdissociated antibodies (for verification of non-specific binding). A reference serum sample added to 6 wells in twofold dilutions (1:50 to 1:1600) was included in each plate. After incubation for $60 \mathrm{~min}$ at $37^{\circ} \mathrm{C}$, the plates were washed three times and $100 \mu \mathrm{L}$ of conjugate goat anti-IgG peroxidase (Sigma Chemical Co, St Louis, MO, USA), diluted 1:6000 in 1\% BSAPBS, was added to each well and incubated at $37^{\circ} \mathrm{C}$ for 1 hour. The amount of bound peroxidase conjugated immunoglobulin was quantitated by reading the absorbance at 450ๆm (A450) using 3,3',5,5'-tetramethylbenzidine (TMB) as a substrate in the presence of hydrogen peroxide. The titres of each sample were determined relative to the reference serum sample included on each plate using single point reference line assay. Thus, dose-response curves were calculated from the absorbance value of the reference serum dilutions on the $\mathrm{Y}$ axis against $2 \log$ reference serum titres on the $\mathrm{X}$ axis. The method of least squares was used to generate a straight line fit [32] to the portion of the curve which was considered linear, if the correlation between A450 and log dilution was $\geq 0.95$. When appropriate, single values considered to deviate too far the straight line, were omitted. For free antibodies, the absorbance value of each sample was transferred to the straight line of the curve to obtain eu/mL. For IC-dissociated antibodies, the absorbance value of each sample was calculated by subtracting the absorbance value of each sample in wells without antigens from the absorbance value of the same sample in wells with the antigen; the difference in absorbance values of each sample was then transferred to the curve to obtain eu/mL.

\section{ELISA with CFA}

The levels of free antibodies were determined as previously described [29]. Serum samples were diluted 1:200 in 1\% skim milk-PBS with the addition of $0.05 \%$ Tween-20. Protein A coupled with horseradish peroxidase (Sigma Chemical Co, St Louis, MO, USA), 2,2'-azino-bis(3-ethylbenzthiazoline-6sulfonic acid) and hydrogen peroxide were used in the process of revealing the immune reaction. The levels of IC-dissociated antibodies against CFA were quantified as described above in Section "Enzyme-immunoassay ELISA with recombinant antigens".

All of the samples were tested in duplicate wells each day and at least twice on different days.

\section{Data Analysis}

Comparison of distribution of antibody titers and frequencies of positivities between groups of patients were made using the Mann-Whitney $U$ test and the $\chi 2$ test, respectively. The significance of differences in frequencies obtained with each pair of tests was studied using McNemar's test. The correlation between the levels of free and complexdissociated antibodies against the same antigen were studied with Pearson Regression Analysis. The level of concordance of humoral response to different antigens in the same patients was assessed using Kappa statistics, a method to correct for chance agreement. Sensitivity and specificity were calculated using standard methods [33].

\section{Results}

Antibody Levels Against the Three Recombinant Antigens and CFA In serum and Dissociated IC

Individual IgG antibody levels against the 38-kDa, Ag16 and Ag85B recombinant antigens and CFA in serum and dissociated IC are presented in the Figure 1. The significance 
Figure 1. Scattergram of individual seroreactivity reflecting levels of IgG antibody against 38-kDa antigen (left upper position, each panel), Ag16 (right upper position, each panel), Ag85B (left lower position, each panel) and culture filtrate antigens (right lower position, each panel) in serum (panel A) and in dissociated immune complexes (panel B) isolated from the following groups of patients: smear-positive pulmonary TB (a), smear-negative pulmonary TB (b), extrapulmonary TB (c), mycobacteriosis (d), mycoses (e),leprosy (f), apparently healthy subjects (g), patients with underlying disorders other than mycobacteriosis, mycoses and leprosy (h). Responses. Hatched horizontal lines represent the cut-off point calculated for each antigen; the significance of the differences between the mean antibody levels of apparently healthy subjects and the mean antibody level of each group are also shown by asterisks (Mann-Whitney U test ;*p<0.05; ${ }^{* *} \mathrm{p}<0.01 ;{ }^{* * *} \mathrm{p}<0.001$ ).

A
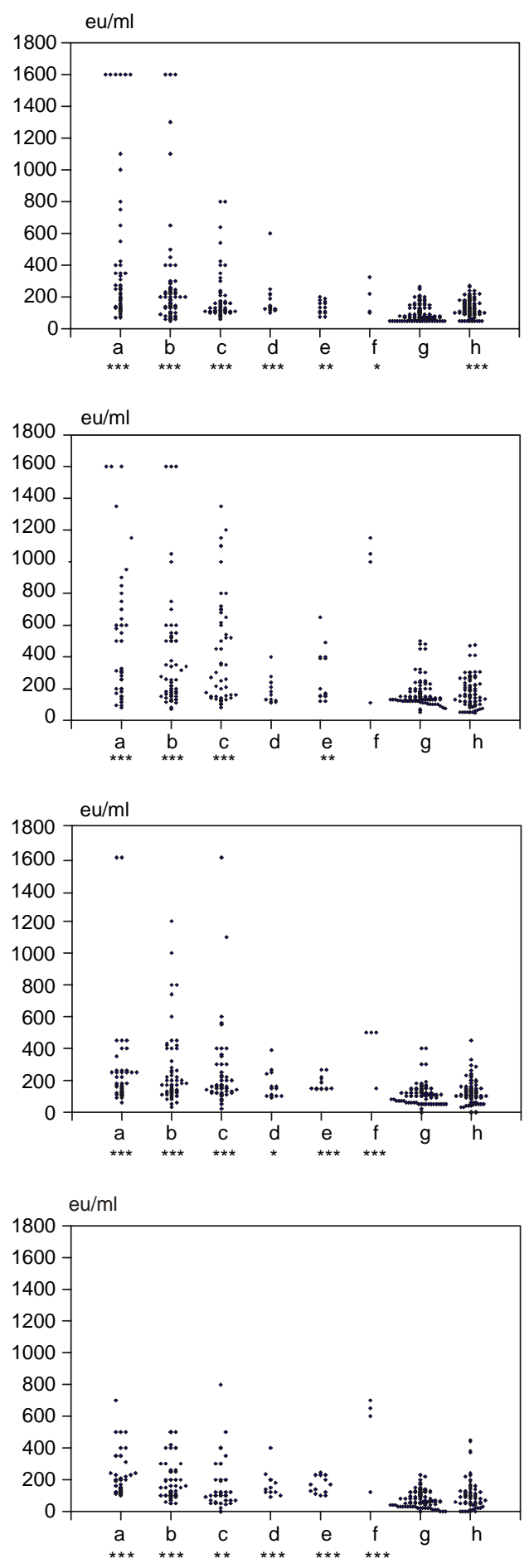

B
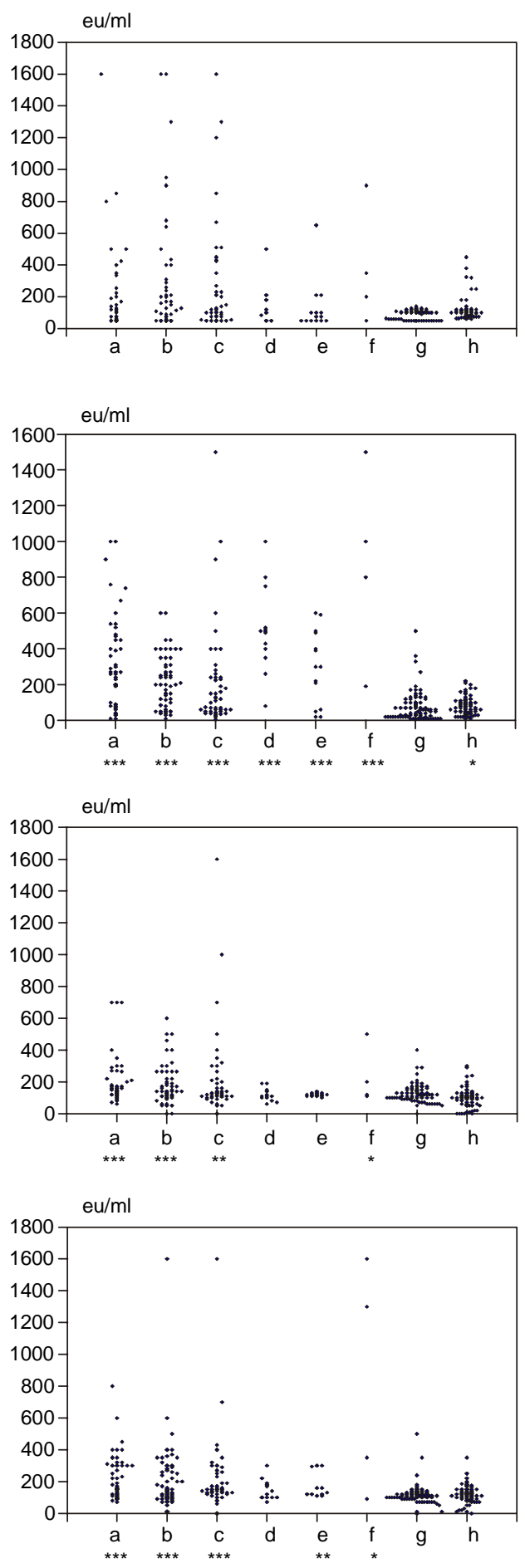


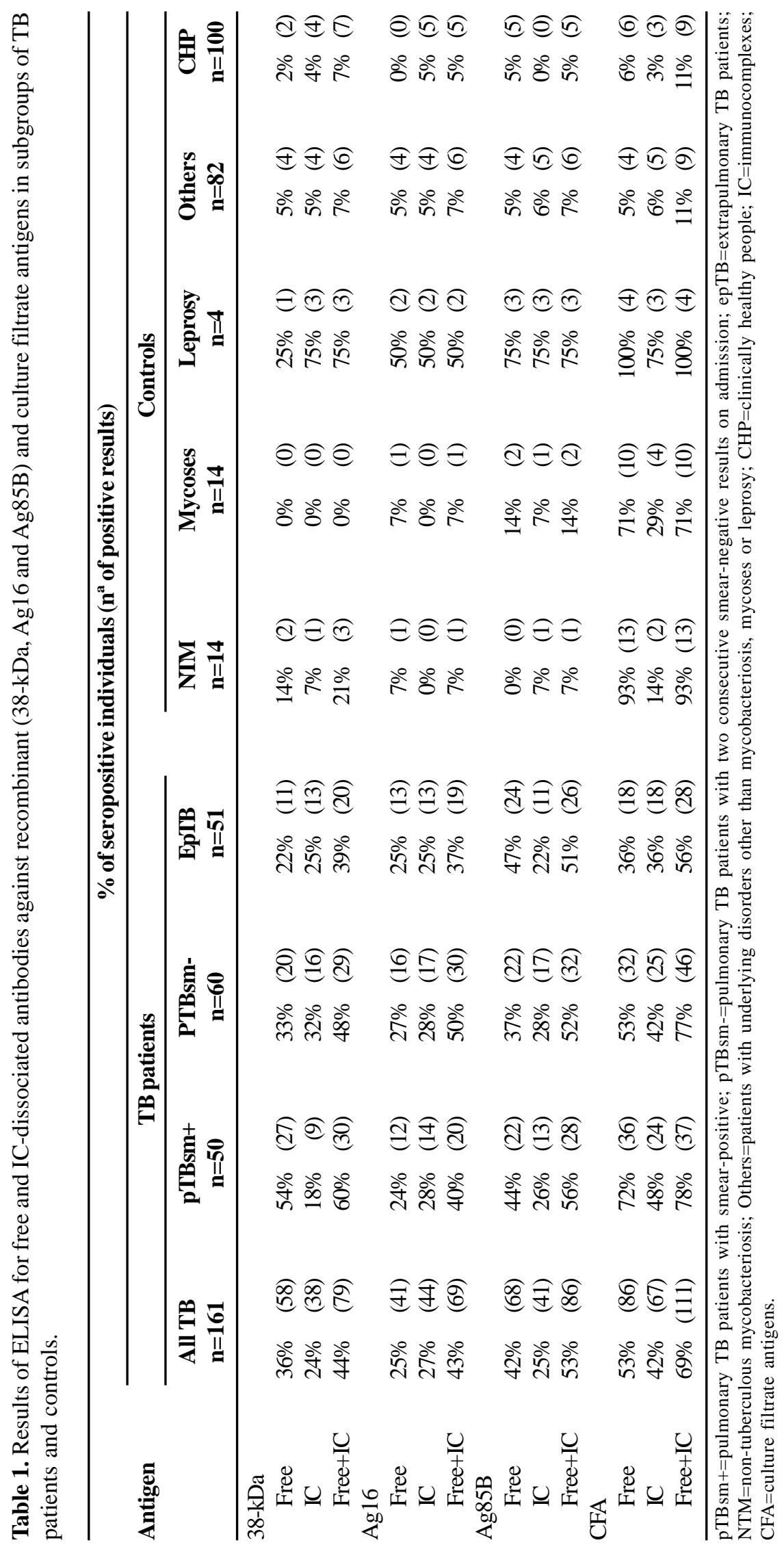


between the antibody levels of the clinically healthy patients group (CHP) and those of each group are also shown.

Among the TB group, we observed that, for the $38-\mathrm{kDa}$ antigen and CFA only, the level of free antibodies in the pTBsm+ group was significantly higher as compared with those found in the pTBsm- and epTB groups (Mann-Whitney U test; $\mathrm{p}<0.05$ ). On the other hand, we found, for Ag85B only, that the IC-dissociated antibody mean level in the pTBsm+ group was significantly higher than those observed for pTBsm- and epTB groups (Mann-Whitney $U$ test, $\mathrm{p}<0.05$ ). The analysis of the correlation between the level of free and complex-dissociated antibodies against each individual recombinant antigen in sera from TB patients showed a slight correlation within these parameters $(\mathrm{r}=0.24, \mathrm{p}=0.03$ for free and complex antibodies anti-38-kDa; $r=0.12, p=0.04$ for free and complex antibodies anti-Ag16; $\mathrm{r}=0.24, \mathrm{p}=0.02$ for free and complex antibodies anti-Ag85B); similar results were obtained when this analysis was made for $\mathrm{CFA}(\mathrm{r}=0.17, \mathrm{p}=0.03)$.

\section{Determination of ELISA Cut-Off}

In previous evaluations of different serological tests for the diagnosis of TB, it has been pointed out that patients with non-tuberculous diseases have shown a more frequent incidence of false-positive results than that observed in healthy people, likely indicating that some diseases (especially acute pneumonia) may cause interferences, possibly owing to disorders in immune response occurring in the course of these diseases [34,35]. In the present work, among the nontuberculous group of patients, the mean levels of free and ICdissociated antibodies obtained from patients with underlying disorders other than TB (Others) were higher than those calculated for CHP, with the exception of the determination of IC-dissociated antibodies against Ag16. Although these differences were only significant for free antibodies against the 38-kDa antigen and CFA, to control for any potential effect, the cut-off for each antigen was calculated from the mean plus 2 standard deviations measured in the group of Others (Figure 1).

\section{ELISA Analysis of Individual Recombinant Antigens and CFA}

Percentages of positive results of ELISA for free and ICdissociated antibodies against recombinant antigens (38-kDa, Ag16 and Ag85B) and CFA in subgroups of TB patients and controls are presented in Table 1. For each of the antigens evaluated, results of ELISA for free and IC-dissociated antibodies are shown both singly and in combination (calculated on the basis of results obtained with single antigens).

In relation to free antibodies, for 38-kDa, significantly higher sensitivities were obtained in pTBsm+ than in pTBsm( $\chi 2$ test, $54 \%$ vs. 33\% for pTBsm+ vs. pTBsm-, respectively, $\mathrm{p}=0.047$ ) and in pTB with respect to epTB ( $\chi 2$ test, $41 \%$ vs. $22 \%$, for pTB and epTB, respectively, $\mathrm{p}=0.015)$. Ag85B was the most efficient in detecting epTB (McNemar's test, $47 \%$ vs. $22 \%$, for Ag85 and 38-kDa, respectively, $\mathrm{p}=0.004 ; 47 \%$ vs. $25 \%$ for Ag85 and Ag16, respectively, p=0.041). For the three recombinant antigens specificity was $95 \%$ in Others, whereas it ranged from $95 \%$ to $100 \%$ in CHP, from $86 \%$ to $100 \%$ in NTM and mycoses and from $25 \%$ to $75 \%$ in leprosy, whereas ELISA with CFA, gave extremely poor levels of specificity, with values of $7 \%$ in NTM, $29 \%$ in mycoses and $25 \%$ in leprosy. By analyzing the concordance of free and IC-dissociated serologic responses against each individual antigen within the same subgroup of TB patients we found that the level of Kappa was never higher than 0.4 , reflecting a high heterogeneity between free and IC-dissociated responses against each of the antigens evaluated. Considering this heterogeneity, it was important to study, for each of the antigens, the contribution of IC-dissociated antibody results to free antibody results. In almost all subgroup of TB patients, for each of the antigens studied, the combination of measuring free and complexdissociated antibodies significantly increased the sensitivity of the tests in comparison with the determination of only free antibodies (McNemar's test, $\mathrm{p}<0.05$, for combination of results for free and IC-dissociated antibodies vs. free antibody results in all subgroups of TB patients, with the exception of pTBsm+ for 38-kDa antigen and epTB for Ag85B) without any significant loss of specificity (McNemar's test, $\mathrm{p}>0.05$, for combination of free and IC-dissociated antibody results $v s$. free antibody results against each of the antigens studied in all control subgroups).

The serological reactivity of a given antigen could be evaluated not only by estimating the number of antibody responders among TB patients but also by determining the proportion of high-level responses among sera reacting with that antigen. To analyze levels of antibody response, we counted the number of highly reactive sera (defined as those sera that have antibody levels greater than or equal to the mean level of "Others group" plus 6 standard deviations) and then calculated the proportion of high-level antibody responses among responders to each antigen. Ag16 elicited high level free antibody responses in more than half of the responders (21 of 41 responders, 51\%), whereas 38-kDa antigen elicited high level antibody responses in $40 \%$ ( 23 of 58 ) of the responders to this antigen. Ag85B elicited strong humoral response in merely about one third (22 of 68) of the responders. Only around a quarter of IC-dissociated antibody responders against 38-kDa and Ag16 (11 of 38 and 11 of 44, for 38-kDa and Ag16, respectively) had high level antibody responses against either antigen, whereas there was almost no high reactive sera against Ag85B (5\%, 2 of 41).

\section{Heterogeneity of Antigen Recognition}

Analysis of antigen recognition by individual sera revealed that the pattern of antigens reacting with serum antibodies varied greatly from patient to patient. No single antigen or group of antigens was reactive with all, or even with most, sera from TB patients. The number of antigens recognized by free antibodies varied markedly from one (54 of the $161 \mathrm{~TB}$ patients) to three ( 15 of the 161 patients). In the same way, the 
Table 2. Concordance of serologic responses within the same TB subjects by diagnostic group.

\begin{tabular}{|c|c|c|c|c|c|c|}
\hline \multirow{3}{*}{$\begin{array}{l}\text { Diagnostic } \\
\text { group }\end{array}$} & \multicolumn{2}{|c|}{ 38-kDa and Ag 16} & \multicolumn{2}{|c|}{ 38-kDa and Ag85B } & \multicolumn{2}{|c|}{ Ag16 and Ag85B } \\
\hline & Карра (95\%СI) & & Карра (95\%CI) & & Карра (95\%СI) & \\
\hline & Free antibodies & IC-dissociated & Free antibodies & IC-dissociated & Free antibodies & IC-dissociated \\
\hline pTBsm+ & $0.15(-0.11-0.41)$ & $0.31(0.05-0.57)$ & $0.43(0.15-0.71)$ & $0.22(0.04-0.48)$ & $0.32(0.08-0.60)$ & $0.44(0.16-0.72)$ \\
\hline pTBsm- & $0.16(-0.10-0.42)$ & $0.49(0.23-0.75)$ & $0.19(-0.07-0.49)$ & $0.45(0.19-0.71)$ & $0.19(-0.05-0.43)$ & $0.42(0.16-0.68)$ \\
\hline ерТВ & $0.35(0.07-0.63)$ & $0.38(0.1-0.66)$ & $0.33(0.09-0.57)$ & $0.34(0.06-0.62)$ & $0.18(-0.08-0.44)$ & $0.46(0.18-0.74)$ \\
\hline
\end{tabular}

Table 3. Results of combination of each individual antigen serological test (cumulative results) and multi-antigen cocktail in subgroups of TB patients and controls.

\begin{tabular}{|c|c|c|c|c|c|c|}
\hline \multirow{2}{*}{$\begin{array}{l}\text { Group of } \\
\text { patients(number } \\
\text { of subjects) }\end{array}$} & \multicolumn{3}{|c|}{ Cumulative results of single antigens ${ }^{\text {a }}$} & \multicolumn{3}{|c|}{ Cocktail $^{\text {b }}$} \\
\hline & Free antibodies & $\begin{array}{l}\text { IC-dissociated } \\
\text { antibodies }\end{array}$ & Free + IC & Free antibodies & $\begin{array}{l}\text { IC-dissociated } \\
\text { antibodies }\end{array}$ & Free + IC \\
\hline \multicolumn{7}{|c|}{ Sensitivity (number of positive results) } \\
\hline All TB(161) & $64 \%(103)$ & $43 \% \quad(69)$ & $76 \%(123)$ & $54 \% \quad(87)$ & $30 \% \quad(48)$ & $63 \%(102)$ \\
\hline PTB sm+ (50) & $74 \% \quad(37)$ & $42 \% \quad(21)$ & $80 \% \quad(40)$ & $64 \% \quad(32)$ & $30 \% \quad(15)$ & $70 \% \quad(35)$ \\
\hline PTBsm- (60) & $62 \% \quad$ (37) & $45 \% \quad(27)$ & $80 \% \quad(48)$ & $50 \% \quad(30)$ & $30 \% \quad(18)$ & $65 \% \quad(39)$ \\
\hline EpTB(51) & $57 \% \quad(29)$ & $41 \% \quad(21)$ & $69 \% \quad(35)$ & $49 \% \quad(25)$ & $29 \% \quad(15)$ & $55 \% \quad(28)$ \\
\hline \multicolumn{7}{|c|}{ Specificity (number of negative results) } \\
\hline All controls (214) & $89 \%(191)$ & $90 \%(192)$ & $83 \%$ (178) & $96 \%(205)$ & $96 \%(206)$ & $95 \%(204)$ \\
\hline NTM(14) & $86 \% \quad(12)$ & $86 \% \quad(12)$ & $76 \% \quad(11)$ & $93 \% \quad(13)$ & $100 \%$ & $93 \% \quad(13)$ \\
\hline Mycoses (14) & $86 \% \quad(12)$ & $93 \% \quad(13)$ & $86 \% \quad(12)$ & $93 \% \quad(13)$ & $100 \%$ & $93 \% \quad(13)$ \\
\hline Leprosy (4) & $25 \%$ & $25 \%$ & $25 \%$ & $25 \%$ & $25 \% \quad(1)$ & $25 \%$ \\
\hline Others (82) & $88 \% \quad(72)$ & $90 \% \quad(74)$ & $83 \% \quad(68)$ & $98 \% \quad(80)$ & $96 \% \quad(79)$ & $96 \% \quad(79)$ \\
\hline CHP (100) & $94 \% \quad(94)$ & $92 \% \quad(92)$ & $86 \% \quad(86)$ & $98 \% \quad(98)$ & $98 \% \quad(98)$ & $98 \% \quad(98)$ \\
\hline
\end{tabular}

a Calculated on the basis of results obtained with single antigens; ${ }^{\text {b }}$ Experimentally obtained by testing multi-antigen cocktail; Cocktail=38kDa + Ag16 + Ag85B; pTBsm+=pulmonary TB patients with smear-positive; pTBsm-=pulmonary TB patients with two consecutive smearnegative results on admission; epTB=extrapulmonary TB patients; NTM=non-tuberculous mycobacteriosis; Others=patients with underlying disorders other than mycobacteriosis, mycoses or leprosy; CHP=clinically healthy people; IC=immunocomplexes.

number of antigens recognized by IC-dissociated antibodies varied from one (29 of 161 patients) to three (14 of 161 patients). Furthermore, by analyzing the concordance of serologic responses of each individual antigen within the same group of TB patients (Table 2), we found that the level of Kappa was never higher than 0.5, reflecting the above mentioned heterogeneous response at each of the antibody levels.

\section{Combination of Different Individual Antigens Assays}

Considering the heterogeneity of free and IC-dissociated antibody responses against each of the antigens, it was important to analyze the combination of results when any one of the antigens gave a positive response in free or ICdissociated antibody assays. The results of combinations are shown in Table 3.

In the TB patient group, the combination of positive results for free antibodies against any of the antigens increased the percentage of positivity of the individual assays from $42 \%$ (for Ag85B, the antigen with the highest individual sensitivity) (Table 1) to $64 \%$ (Table 3); the highest increase in sensitivity was obtained in the pTBsm- group in which the sensitivity improved at least 25\% (from 37\% for Ag85B to 62\% after the combination of different individual assays). The contribution of measuring IC-dissociated antibodies also increased de diagnostic utility, but this contribution was only significantly for pTBsm- (from $62 \%$ after the combination of measuring free antibodies against any of the antigens to $80 \%$ after combining the results of measuring IC-dissociated antibodies against any of the antigens, McNemar's test, $\mathrm{p}=0.001$,) and for epTB (from 57\% after the combination of measuring free antibodies against any of the antigens to $69 \%$ after combining the results of measuring IC-dissociated antibodies against any of the antigens, McNemar's test, $\mathrm{p}=0.031$ ) (Table 3).

On the other hand, the combination of individual results increased the false positive rate, especially in the group of Others, in which the specificity dropped from $95 \%$ for free antibody assays to each of the recombinant antigens, to 88\%, after the combination of positive results of free antibody assays to any of the individual antigens, and to 83\% after considering the contribution of any positive result obtained by measuring IC-dissociated antibodies against any of the antigens (95\% vs. 83\% for free antibody assays with each antigen and for combination of free and complexdissociated results against any of the antigens, respectively, McNemar's test, $\mathrm{p}=0.002$ ) (Table 3). 


\section{ELISA Analysis of Multi-Antigen Cocktails}

Besides the combination of single antigen results, the development of multi-antigen cocktails for the serodiagnosis of TB is currently seen as a way of improving the diagnostic utility of this method. Therefore, we studied the serological activity of a three-antigen cocktail (Table 3). The sensitivity of the cocktail was lower than that expected on the basis of single antigen results. Only 87 of the expected 103 reactors in the free antibodies assays and 48 of the expected 69 reactors in the IC-dissociated antibody assays were positive with the cocktail, comprising a loss of $10 \%$ and $13 \%$ sensitivity for free and IC-dissociated assays, respectively. We observed that some low level reactivities to one or more single antigens were lost when using cocktails. This observation was also detected in samples from controls, resulting in a gain of diagnostic specificity, both for free and IC-dissociated assays. The contribution of IC-dissociated antibody results to the free-antibody results against the cocktail was significant for the diagnosis of pTBsm- patients, with a gain in sensitivity of 15\% (McNemar's test, $\mathrm{p}=0.04$ ) (Table 3). Interestingly, even after combining results of free and IC-dissociated antibody anti-cocktail, the level of false positive results in the controls was low, resulting in specificity values ranging from $93 \%$ to $98 \%$ in the different control subgroups, with the exception of the leprosy group.

\section{Discussion}

Because of their ease of performance and costeffectiveness, serodiagnostic tests constitute a promise for early detection of TB. In earlier days, these tests were attempted using semi-purified antigens. However, because these antigens contain cross-reactive epitopes, they yielded reactions of poor specificity. Many authors have reported that the use of purified antigens has partially solved this problem by obtaining high specificity in spite of the reduced sensitivity of each single serological assay [1,3]. Our study confirms these previous observations. In fact, the use of single recombinant antigens such as Ag85B, 38-kDa, Ag16 for the detection of free antibodies allows a higher specificity than an assay based on culture filtrate antigens, especially with the group of mycobacteriosis and mycoses, but at the cost of a low level of sensitivity. This lack of antibody response in a large proportion of patients has been attributed mainly to two factors: (i) the possible formation and circulation of specific immune complexes that are not detected by regular ELISA performed with whole serum and (ii) the person-to-person variation of antigen recognition. A number of methods have been used for detection and quantification of IC in various chronic diseases [36]. Since Bhattacharya et al.. [37], Singh et al. [38] and Bajaj et al. [39] reported a significant rise in IC levels when using PEG precipitation in TB patients, this method has been the one of choice of several studies. In our work, the levels of complex IgG were determined using PEG precipitation method, but with the addition of a process of dissociation of IC by treating them with acid $\mathrm{pH}$; Udaykumar and Saxena [40] have shown that this acid $\mathrm{pH}$ procedure induces higher antigen binding ability of anti- mycobacterial antibodies, present in complex form in TB sera. As they also noticed that antibodies became stickier after exposure to acid $\mathrm{pH}$, and this resulted in higher background absorbance values in ELISA, therefore in order to avoid the measurement of nonspecific binding, for each serum, we subtracted this background increase before using the data for statistical evaluation.

In our work, using a complex antigen, such as CFA, we found values of IC-dissociated IgG positivity ranging from $36 \%$ to $48 \%$ among the different subgroups of TB patients. Accordingly, an early study [40], using a similar antigen, revealed that about $40 \%$ of the TB patients had positive results to IC-dissociated IgG. Overall, we found a sensitivity for free antibody anti-CFA of about 40\% and 50\% in epTB and smearnegative pTB, respectively, whereas it increased to $60 \%$ and $80 \%$ in epTB and smear-negative pTB, after adding the contribution of positive results for IC-dissociated antibody anti-CFA. However, the levels of specificity were poor, especially in the group of diseases considered in the differential diagnosis of TB, such as mycobacteriosis and mycoses. On the other hand, using individual recombinant antigens, our results showed a sensitivity ranging from $22 \%$ to $54 \%$ when measuring free IgG antibodies in the different subgroups of TB patients; similar low levels of positivity were obtained by Lyashchenko et al. [39] when determining serum IgG antibodies against the same group of recombinant antigens; we noticed a variable contribution of complex IgG antibody to the diagnostic efficacy of the ELISAs performed with serum, depending on the tuberculosis state and the individual antigen used. Overall, the cumulative determination of free and complex-dissociated antibodies against each individual antigen showed a sensitivity of up to around 55\% of TB cases. The best improvement in sensitivity was seen in paucibacillary pTB patients. Previous reports [42-44], using chromatography purified 38-kDa, Ag16 and Ag85B antigens, found that detection of antibodies in IC, in addition to free antibodies, greatly improved the outcome of each individual antigen test, with high levels of sensitivity (near $85 \%$ in cultureproven cases and $70 \%$ in roentgenogram cases) and specificity. Studies on reactivity of human and murine sera with antigens of $M$. tuberculosis suggest that, in contrast to the murine antibodies, human antibodies elicited during natural disease progression would recognize glycosylated, conformational epitopes on native proteins [26,45]. In addition, another report showed that the reactivity of human sera with overlapping peptides of Ag16 was 20 to 50\% lower than the reactivity with native antigens [46]. Since recombinant antigens used in this study were expressed in $E$. coli, the low reactivity of sera with these proteins may be due to the lack of glycosylation. This factor would contribute to explaining the decreased reactivity observed with each individual recombinant antigen in this study in comparison with the results obtained by Uma Devy et al. [42] and Raja et al. [43,44]

www.bjid.com.br 
with native proteins. Another issue related to the decreased reactivity of our antigens may be the existence of an association between antibody titers against particular $M$. tuberculosisspecific epitopes and certain alleles, so that the performance of the same assays on populations from different ethnic origins may give contrasting results. Genetic restriction of immune response to mycobacteria [47] and/or differential characteristics among mycobacterial strains prevalent in distinct regions of the world, inducing different host responses, could account for these differences. Accordingly, evaluation of a commercial kit based on the determination of antibodies anti r38kDa and Ag16 gave contrasting results when it was used on population from different ethnic origins [48-50].

Our data raise the issue of the variability of the antimycobacterial antibody response in different individuals and cohorts, and highlight the difficulties of identifying a single recombinant mycobacterial antigen to develop serologic tests of wide applicability. The number of serologically reactive antigens varies greatly from individual to individual. In a given sera the level of specific antibodies also varies with the antigen, irrespective of the total number of antigens recognized by that particular serum, and this heterogeneity was seen not only for free antibodies but also for those in IC. This heterogeneous humoral response found using proteinaceous antigens provide the rational for the development of a multiantigen test. Therefore, we studied the serological activity of a three-recombinant antigen cocktail. In agreement with Gennaro [51], our work showed that high level serum reactivities to a single antigen are sufficient to yield diagnostic positivity to cocktails of multiple antigens. Some low-level reactivities in sera from tuberculosis and control patients to one or more antigens are lost when using cocktails; therefore false-positive reactions caused by low level reactivities in tuberculosis-negative sera were lost when a multi-antigen cocktail was used, with a gain in diagnostic specificity (Table 3). However, there is also a loss of diagnostic sensitivity associated with low-level reactivities in sera for tuberculous patients. This finding could, presumably, be due to antigens interfering with each other in the microtiter plate for binding to polystyrene.

In this respect, we found that Ag16, although having the poorest sensitivity of the evaluated antigens, because most of its reactivity was of high-level, its role in the positivity of the cocktail ELISA was high; on the other hand, Ag85B, that showed the highest level of sensitivity, as most of its serological responses were of low-level, its contribution to the sensitivity of the cocktail assay was markedly lower than that expected.

Although patients who are smear-negative clearly expel fewer organisms than those who are smear-positive, the delay in treatment and lack of isolation may significantly contribute to the propagation of TB [52]. Measures to accelerate the rapid diagnosis of these patients may contribute to controlling TB. Our results show that in HIV-negative, smear-negative
pTB patients, free and complex-dissociated antibodies to each individual 38-kDa, Ag16 and 85B antigens are detected only in a small proportion of patients; combining in the same plate the reactivity of all three antigens raised the sensitivity to about 65\%, with a very good level of specificity, especially in patients included in the differential diagnosis of TB such as patients with mycoses and mycobacteriosis.

Only a limited number of studies have been conducted to measure free and complex-dissociated antibodies in sera from patients with epTB. Our data showed that even after combination of free and complex-dissociated antibodies to 38-kDa, Ag16 and 85B antigens, epTB can be diagnosed with only 55\% sensitivity. Accordingly, Simmoney et al. [53], in their evaluation of the levels of antibodies to glycolipid antigens, also found that the contributive effect of complexed IgG specific antibodies was restricted, almost entirely, to children with pTB, and to a lesser extent those with epTB. In tuberculous meningitis, it has been suggested that antigenantibody complexes formed in the central nervous system could be unable to cross the blood brain barrier and may be responsible for false negative results found in patients with meningitis in some trials [54]; the antigen-antibody formed in the cerebrospinal fluid (CSF) may be present in CSF in the active disease but may not be found in serum. In accordance with this hypothesis, in the four cases of culture proven tuberculous meningitis, enzymatic units for the detection of antibodies in ICs were below the cut-off point. Further, these patients responded to specific antituberculosis therapy, thus ruling out the possibility of false negativity.

With both free and complex-dissociated antibodies to the cocktail of antigens, positive (false positive) results were obtained in 10 of 214 controls. The maximum number of false positive results (75\%) was obtained in patients with leprosy, which correlates with a previous report [50] in which three of four patients with leprosy were positive for antibodies anti recombinant 38- and 16-kDa. It is well known that the inclusion of patients with non-TB underlying disorders is crucial for clinical evaluation of new tests, since they are at risk of being confused with TB patients and an unspecific hyperglobulinemia described for some non-respiratory diseases may contribute to false positive results [55]; for this reason, in the present work, we have included a large number of non-TB disease patients (38\% with respect to the overall controls). In this group, eighty two patients with suspected pulmonary tuberculosis gained a firm alternative diagnosis, but three (4\%) were positive for either free or complex antibodies to the cocktail. The decision to treat patients for tuberculosis in whom there was no microbiological confirmation of disease was clearly made on clinical grounds. Thus, in these patients, there must be at least one symptom or sign of TB in addition to an abnormal chest radiograph. Antibody titers $>400 \mathrm{eu} / \mathrm{mL}$ and $>350 \mathrm{eu} / \mathrm{mL}$ for free and complex antibody anti-cocktail assays, respectively, were not found when screening patients with other underlying disorders (including mycoses and mycobacteriosis), and this antibody level could be valuable in deciding which 
patients without bacteriological confirmation of tuberculosis should be treated. Because a considerable number of tuberculous patients have free or complex antibody levels between the cut off point $(200 \mathrm{eu} / \mathrm{mL}$ and $250 \mathrm{eu} / \mathrm{mL}$ for free and complex antibody anti-cocktail, respectively) and those indicated above, patients included in this range merit continued observation.

To our knowledge, this is the first report that describes the performance of measuring both free and complexdissociated antibodies against recombinant proteins of $M$. tuberculosis in a broad range of tuberculous patients including epTB. We have found that no single recombinant antigen was reactive with most sera from TB patients even after the measurement of both free and complex-dissociated antibodies; the use of multi-antigen cocktails improved the diagnostic utility of the ELISA assay, allowing the identification of almost $70 \%$ of smear negative pTB, with a high level of specificity especially in the group of patients with diseases included in the differential diagnosis of $\mathrm{TB}$, such as mycobacteriosis and mycoses; the use of additional recombinant specific antigens of $M$. tuberculosis could lead to the detection of specific free and complex antibodies in almost all patients with active TB. Furthermore, the fact that high level serum reactivities to a single antigen are sufficient to yield diagnostic positivity to cocktails of multiple antigens whereas some low-level reactivities are lost when using cocktails, has important practical implications for the design of antigen combinations that would achieve high diagnostic accuracy; therefore, for a better characterization of a given antigen, it would be very valuable if reports about the reactivity of an antigen would estimate not only the number of antibody responders but also the proportion of high-level responders among sera reacting with that antigen.

\section{References}

1. Bothamley G.H. Serological diagnosis of tuberculosis. Eur Respir J 1995; 20:676s-688s.

2. Chan E.D., Heifets L., Iseman M.D. Immunological diagnosis of tuberculosis: a review. Tuberc Lung Dis 2000;80:131-40.

3. Lyashchenko K.P., Singh M., Colangeli R., Gennaro M.L. A multiantigen print immunoassay for the development of serological diagnosis of infectious diseases. J Immunol Methods 2000;242:91-100.

4. Brostoff J., Lenzini L., Rottoli P. Immune complexes in the spectrum of tuberculosis. Tubercle 1981;62:169-73.

5. Carr R.I., Chakraborty A.K., Brunda M.J., et al. Immune complexes and antibodies to BCG in sera from patients with mycobacterial infections. Clin Exp Immunol 1980;39:562-9.

6. Grange J.M. The humoral immune response in tuberculosis: its nature, biological role and diagnosis usefulness. Adv Tuberc Res (Kargel, Basel) 1984;21:1-78.

7. Daniel T.M., Anderson P.A. The isolation by immunoabsorbent affinity chromatography and physicochemical characterization of Mycobacterium tuberculosis antigen 5. Am Rev Respir Dis 1978; $117: 533-9$.

8. Coates A.R.M., Hewit J., Allen B.W., Ivanyi J. Antigenic diversity of Mycobacterium tuberculosis and Mycobacterium bovis detected by means of monoclonal antibodies. Lancet 1981; $11: 167-9$
9. Benjamin R.G., Daniel T.M. Serodiagnosis of tuberculosis using the enzyme-linked immunoabsorbent assay (ELISA) of antibody to Mycobacterium tuberculosis antigen 5. Am Rev Respir Dis 1982;126:1013-16.

10. Balestrino E.A., Daniel T.M., de Latini M.D., et al. Serodiagnosis of pulmonary tuberculosis in Argentina by enzyme-linked immunosorbent assay (ELISA) of IgG antibody to Mycobacterium tuberculosis antigen 5 and tuberculin purified protein derivative. Bull World Health Organ 1984;62:755-61.

11. Daniel T.M., Benjamin R.G., Debanne S.M., et al. ELISA of IgG antibody to $\mathrm{M}$. tuberculosis antigen 5 for serodiagnosis of tuberculosis. Indian J Pediatr 1985;52:349-55.

12. Daniel T.M., De Murillo G.L., Sawyer J.A., et al. Field evaluation of enzyme-linked immunosorbent assay for the serodiagnosis of tuberculosis.Am Rev Respir Dis 1986;134:662-5.

13. Ma Y., Wang Y.M., Daniel T.M. Enzyme-linked immunosorbent assay using Mycobacterium tuberculosis antigen 5 for the diagnosis of pulmonary tuberculosis in China. Am Rev Respir Dis 1986; $134: 1273-5$.

14. Cole R.A., Lu H.M., Shi Y.Z., et al. Clinical evaluation of a rapid immunochromatographic assay based on the $38 \mathrm{kDa}$ antigen of Mycobacterium tuberculosis on patients with pulmonary tuberculosis in China. Tuber Lung Dis 1996;77:363-8.

15. Chiang I.H., Suo J., Bai K.J., et al. Serodiagnosis of tuberculosis. A study comparing three specific mycobacterial antigens. Am J Respir Crit Care Med 1997; 156:906-11.

16. Verbon A., Hartskeerl R.A., Schuitema A., et al. The 14,000molecular-weight antigen of Mycobacterium tuberculosis is related to the alpha-crystallin family of low-molecular-weight heat shock proteins. J Bacteriol 1992;174:1352-99.

17. American Thoracic Society. Future research in tuberculosis: prospects and priorities for elimination. Am Rev Respir Dis 1986; $134: 401-20$.

18. Bothamley G.H., Beck J.S., Potts R.C., et al. Specificity of antibodies and tuberculin response after occupational exposure to tuberculosis. J Infect Dis 1992;166:182-6.

19. Wiker H.G., Harboe M. The antigen 85 complex: a major secretion product of Mycobacterium tuberculosis. Microbiol Rev 1992;56:648-61.

20. Samanich K., Belisle J.T., Laal S. Homogeneity of antibody responses in tuberculosis patients. Infect Immun 2001;69:4600-9.

21. Sanchez-Rodriguez C., Estrada-Chavez C., Garcia-Vigil J., et al. An IgG antibody response to the antigen 85 complex is associated with good outcome in Mexican Totonaca Indians with pulmonary tuberculosis. Int J Tuberc Lung Dis 2002;6:706-12.

22. Rinke de Wit T.F., Bekelie S., Osland A., et al. The Mycobacterium leprae antigen 85 complex gene family: identification of the genes for the $85 \mathrm{~A}, 85 \mathrm{C}$, and related MPT51 proteins. Infect Immun 1993;61:3642-7.

23. Van Vooren J.P., Drowart A., De Cock M., et al. Humoral immune response of tuberculous patients against the three components of the Mycobacterium bovis BCG 85 complex separated by isoelectric fucosing. J Clin Microbiol 1991;129:2348-50.

24. Lim J.H., Park J.K., Jo E.K., et al. Purification and immunoreactivity of three components from the 30/32kilodalton antigen 85 complex in Mycobacterium tuberculosis. Infect Immun 1999;67:6187-90.

25. Laal S., Samanich K.M., Sonnenberg M.G., et al. Human humoral response to antigens of Mycobacterium tuberculosis: immunodominance of high molecular weight antigens. Clin Diag Lab Immunol 1996;4:49-56.

26. Samanich K.M., Belisle J.T., Sonnenberg M.G., et al. Delineation of human antibody responses to culture filtrate antigens of Mycobacterium tuberculosis. J Infect Dis 1998;178:1534-8.

27. Enarson D.A., Rieder H.L., Arnadottir T., Trebuck A. Manejo de la tuberculosis. Guía para los países de escasos recursos. Paris: International Union against Tuberculosis and Lung Disease, 2000 . 
28. Ministerio de Salud de la República Argentina. Normas técnicas del Programa Nacional de Control de Tuberculosis. Santa Fe, Argentina: Ministerio de Salud de la República Argentina, 2000.

29. Barrera L., Ritacco V., Eisele C., et al. Evaluation of an enzyme immunoassay for the rapid diagnosis of paucibacillary tuberculosis in adults. Medicina (B. Aires) 1989;49:561-6.

30. Hogarth P.J., Logan K.E., Vordermeier H.M., et al. Protective immunity against Mycobacterium bovis induced by vaccination with RV3109ca member of the esat-6 gene family. Vaccine 2005;23:2557-64.

31. Yamashita J.T., Cruaud P., Papa F., et al. Circulating immune complexes in leprosy sera: demonstration of antibodies against mycobacterial glycolipidic antigens in isolated immune complexes. Int J Lepr Other Mycobact Dis 1993;61:44-50.

32. Draper N., Smith H. Applied regression analysis, 2 edn. New York: Wiley, 1981.

33. Toman K. Sensitivity, specificity and predictive value of diagnostic tests. Bull Int Union Tuberc 1979; 54:275-6.

34. Cocito C.G. Properties of the mycobacterial antigen complex A60 and its applications to the diagnosis and prognosis of tuberculosis. Chest 1991;100:1687-93.

35. Alifano M., De Pascalis R., Sofia M., et al. Evaluation of IgA-mediated humoral immune response against the mycobacterial antigen P-90 in diagnosis of pulmonary tuberculosis. Chest 1997;111:601-5.

36. Lambert, P.H., Dixon, F.J., Zubler, et al. A who collaborative study for the evaluation of eighteen methods for detecting immune complexes in serum. J Clin Lab Immunol 1978;1:1-15.

37. Bhattacharya A., Ranadive S.N., Kale M., Bhattacharya S. Antibody-based enzyme-linked immunosorbent assay for determination of immune complexes in clinical tuberculosis. Am Rev Respir Dis 1986;134:205-9.

38. Singh G., Bhau L.N., Saxena S.N. Circulating immune complexes in pulmonary tuberculosis. Indian J Med Res 1986;83:117-22.

39. Bajaj G., Rattan A., Ahmad P. Circulating immune complexes in tuberculosis-an indicator of activity. Indian $\mathrm{J}$ Pediatr 1990;57:203-7.

40. Udaykumar, Saxena R.K. Acid pH-induced changes in the immunoreactivity of specific antigen and antibody in circulating immune complexes from tuberculosis sera. J Clin Lab Anal 1992;6:194-200.

41. Lyashchenko K., Colangeli R., Houde M., et al. Heterogeneous antibody responses in tuberculosis. Infect Immun 1998;66:3936-40.

42. Uma Devi K.R., Ramalingam B., Brennan P.J., et al. Specific and early detection of IgG and IgM antibodies to Mycobacterium tuberculosis $38 \mathrm{kDa}$ antigen in pulmonary tuberculosis. Tuberculosis 2001;81:249-53.
43. Raja A., Uma Devi K.R., Ramalingam B., Brennan P.J. Immunoglobulin $\mathrm{G}$, $\mathrm{A}$ and $\mathrm{M}$ responses in serum and circulating immune complexes elicited by 16 -Kilodalton antigens of Mycobacterium tuberculosis. Clin Diagnostic Laboratory Immunol 2002;9:308-12.

44. Raja A., Uma Devi K.R., Ramalingam B., Brennan P.J. Improved diagnosis of pulmonary tuberculosis by detection of free and immune complex bound anti-30kDa antibodies. Diagn Microbiol Infect Dis 2004;50:253-9.

45. Saxena U., Saxena R.K. Antigenic epitopes on Mycobacterium tuberculosis recognized by antibodies in tuberculosis and mouse antisera. FEMS Microbiol 1991;76:7-12.

46. Verbon A. Development of a serological test for tuberculosis. Trop Geog Med 1994;46:275-9.

47. de Vries R.R., Ottenhoff T.H., van Schooten W.C. Human leukocyte antigens (HLA) and mycobacterial disease. Springer Semin Immunopathol 1988;10:305-18.

48. Wilkinson R.J., Hasløv K., Rappuoli R., et al. Evaluation of the recombinant 38-kilodalton antigen of Mycobacterium tuberculosis as a potential immunodiagnostic reagent. J Clin Microbiol 1997;35:553-57.

49. Pottumarthy S., Wells V.C., Morris A.J. A comparison of seven tests for serological diagnosis of tuberculosis. J Clin Microbiol 2000;38:2227-31.

50. Imaz M.S., Comini M.A., Zerbini E., et al. Evaluation of commercial enzyme-linked immunosorbent assay kits for detection of tuberculosis in Argentinean population. J Clin Microbiol 2004;42:884-7.

51. Gennaro M.L. Immunologic diagnosis of tuberculosis. Clin Infect Dis 2000;30 Suppl 3:243s-6s.

52. Behr M.A., Warren S.A., Salamon H., et al.Transmission of Mycobacterium tuberculosis from patients smear-negative for acid-fast bacilli. Lancet 1999;353:444-9.

53. Simmoney N., Bourrillon A., Lagrange P.H. Analysis of circulating immune complexes (CICs) in childhood tuberculosis: levels of specific antibodies to glycolipid antigens and relationship with serum antibodies. Int J Tuberc Lung Dis 2000;4:152-60.

54. Gupta I., Jain A., Singh N.B., et al. Role of antigen specific circulating immune complexes in diagnosis of tuberculosis. Int $\mathrm{J}$ Tuberc Lung Dis 1998;2:456-61.

55. Bothamley G.H., Rudd R.M. Clinical evaluation of a serological assay using a monoclonal antibody (TB72) to the $38 \mathrm{kDa}$ antigen of Mycobacterium tuberculosis. Eur Respir J 1994; $7: 240-6$. 